\title{
Improving Users' Mental Model with Attention-directed Counterfactual Edits
}

\author{
Kamran Alipour ${ }^{1}$, Arijit Ray ${ }^{2}$, Xiao Lin², Michael Cogswell ${ }^{2}$, Jurgen Schulze ${ }^{1}$, Yi Yao ${ }^{2}$, \\ and Giedrius Burachas ${ }^{2}$ \\ ${ }^{1}$ University of California San Diego \\ ${ }^{2}$ SRI International
}

June 25, 2021

\begin{abstract}
In the domain of Visual Question Answering (VQA), studies have shown improvement in users' mental model of the VQA system when they are exposed to examples of how these systems answer certain Image-Question (IQ) pairs. In this work, we show that showing controlled counterfactual image-question examples are more effective at improving the mental model of users as compared to simply showing random examples. We compare a generative approach and a retrieval-based approach to show counterfactual examples. We use recent advances in generative adversarial networks (GANs) to generate counterfactual images by deleting and inpainting certain regions of interest in the image. We then expose users to changes in the VQA system's answer on those altered images. To select the region of interest for inpainting, we experiment with using both human-annotated attention maps and a fully automatic method that uses the VQA system's attention values. Finally, we test the user's mental model by asking them to predict the model's performance on a test counterfactual image. We note an overall improvement in users' accuracy to predict answer change when shown counterfactual explanations. While realistic retrieved counterfactuals obviously are the most effective at improving the mental model, we show that a generative approach can also be equally effective.
\end{abstract}

\section{Hosted file}

main_text.zip available at https://authorea.com/users/422041/articles/527829-improving-usersmental-model-with-attention-directed-counterfactual-edits

\section{Hosted file}

main.pdf available at https://authorea.com/users/422041/articles/527829-improving-usersmental-model-with-attention-directed-counterfactual-edits

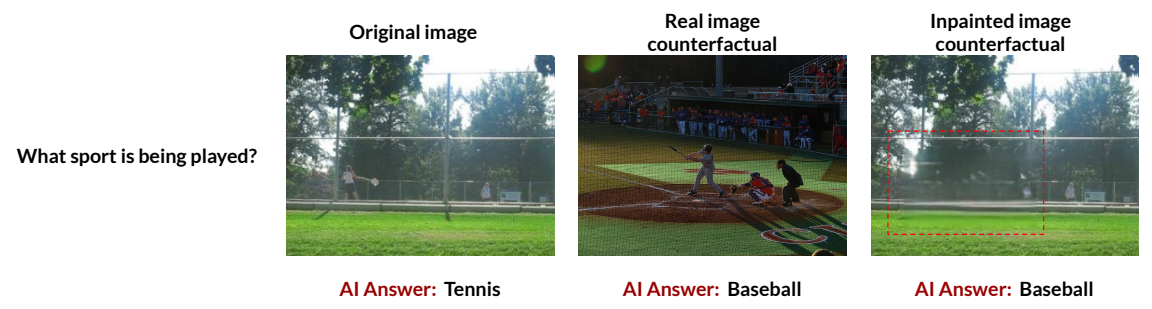




\section{Human Attention Human-based counterfactuals}

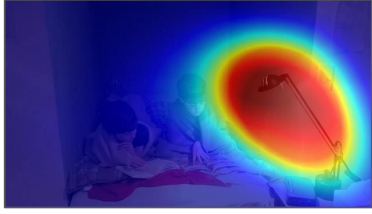

Question: Is the lamp on?

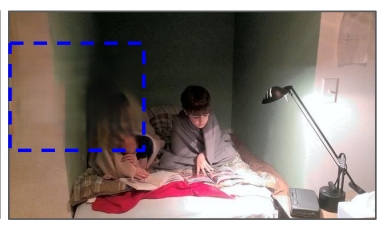

Min. Att.

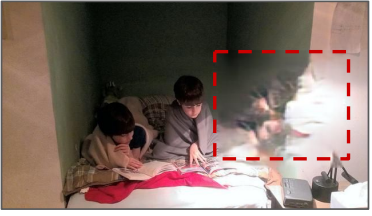

Max. Att.

Baseline: No explanations Inpainted counterfactuals
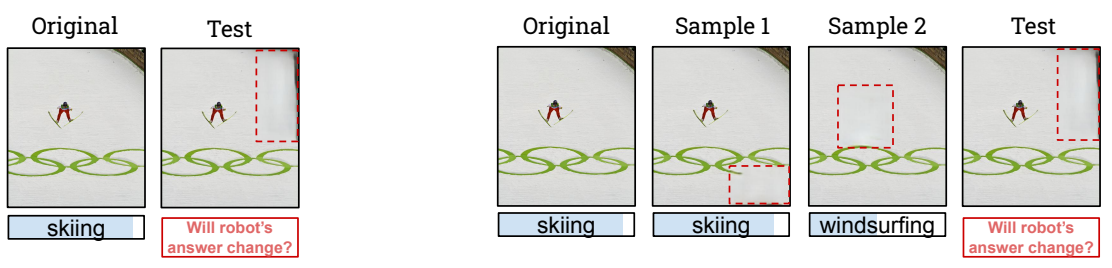

Question: What competitive event is this?

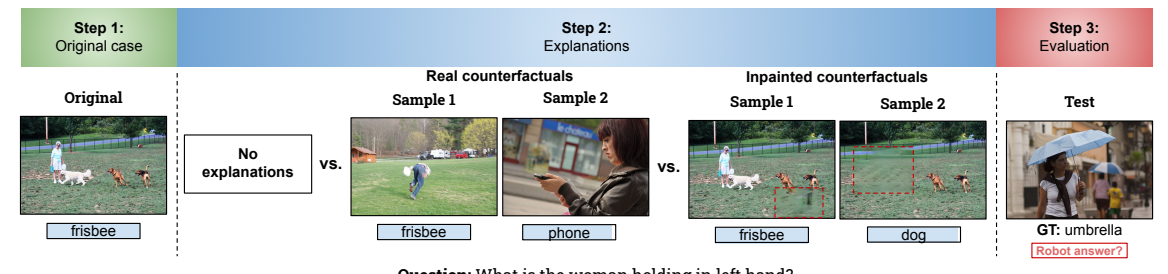

Question: What is the woman holding in left hand? 